\title{
ON CONVERGENCE CLASSES OF SETS
}

\section{GILBERT HELMBERG}

Let $X$ be a topological space (no separation axioms are assumed) and let $\mathfrak{X}$ be an arbitrary set of subsets of $X$. The aim of this paper is to show that if we introduce in $\mathfrak{X}$ a notion of "convergence," having certain relatively weak properties in common with the notion of convergence in $\exp X$ studied by Mrovka [4] and Frolík [1], then the two notions must coincide. Doing this, we shall also study to some extent the duality becoming apparent in statements involving the (closed) topological lim inf and lim sup of a net of sets and exhibit some further properties of the class of nets converging in the sense of $[4 ; 1]$. The author gratefully acknowledges several inspiring discussions he had on this subject with Dr. Grimeisen who has a paper on related problems in the process of being published.

In what follows we shall adhere to the terminology used in [5] and [1] except for some differences in notation that are stated explicitly. Let $X$ be the class of all nets in $\mathfrak{X}$. If $\mathfrak{N} \in \mathfrak{X}$, then we shall write $\mathfrak{N}^{\prime}<\mathfrak{N}$ for the statement: “ $\mathfrak{N}^{\prime}$ is a subnet of $\mathfrak{N}$." Our results are based on the following two lemmas. The first lemma is taken from the proof of $[1$, Theorem 2.12]. A proof of the second one is contained implicitly in the proofs of [1, Theorem 2.7] and [2, Hilfssatz 9].

Lemma 1. Let $\mathfrak{R} \in \mathfrak{X}$ and let $x \notin \lim$ inf $\mathfrak{N}$. Then there is a subnet $\mathfrak{N}^{\prime}<\mathfrak{N}$ such that $x \notin \lim \sup \mathfrak{N}^{\prime}$.

Lemma 2. Let $\mathfrak{N} \in \mathfrak{X}$ and let $x \in \lim$ sup $\mathfrak{N}$. Then there is a subnet $\mathfrak{N}^{\prime}<\mathfrak{N}$ such that $x \in \lim$ inf $\mathfrak{N}^{\prime}$.

We shall combine these two lemmas by formulating Lemma 3 , the two statements of which being equivalent to the corresponding lemmas (the first one also coinciding with [1, Theorem 2.7]). Recall that lim inf $\mathfrak{N} \subset \lim$ inf $\mathfrak{N}^{\prime} \subset \lim$ sup $\mathfrak{N}^{\prime} \subset \lim$ sup $\mathfrak{N}$ for $\mathfrak{N}^{\prime}<\mathfrak{N} \in \mathfrak{X}$.

Lemma 3. For all $\mathfrak{N} \in \mathfrak{X}$

$$
\begin{aligned}
\lim \inf \mathfrak{N} & =\bigcap_{\mathfrak{N}^{\prime}<\mathfrak{N}} \lim \sup \mathfrak{N}^{\prime} \\
\lim \sup \mathfrak{N} & =\bigcup_{\mathfrak{N}^{\prime}<\mathfrak{N}}^{\bigcup} \lim \inf \mathfrak{N}^{\prime} .
\end{aligned}
$$

There are three reasons for using Lemma 3 rather than Lemmas 1

Received by the editors October 12, 1961. 
and 2: 1 . It comes in handier for use. 2. It points out the duality with which we shall be concerned in the sequel. 3 . All the results of the following discussions remain true if we replace $\mathfrak{X}$ by any complete lattice for which Lemma 3 holds as an additional axiom, lim inf and lim sup being then defined for a net $\mathfrak{N}=\left\{A_{d}: d \in D\right\}$ in $\mathfrak{X}$ by $\lim$ inf $\mathfrak{N}$ $=\bigcup_{d_{0} \in D} \bigcap_{d>d_{0}} A_{d}$ and lim sup $\mathfrak{R}=\bigcap_{d_{0} \in D} \bigcup_{d>d_{0}} A_{d}$. It is easy to verify that the remark preceding Lemma 3 holds for any complete lattice. However, Lemma 3 is not true for complete lattices in general. Consider the lattice consisting of the elements $A_{n}(n=1,2, \cdots)$ and of the elements $1=A_{i} \cup A_{j}$ and $0=A_{i} \cap A_{j}$ for $i \neq j$. Let $\mathfrak{R}$ be the sequence $\left\{A_{1}, A_{2}, \cdots\right\}$. Then lim inf $\mathfrak{N}^{\prime}=0$ and $\lim \sup \mathfrak{N}^{\prime}=1$ for all subnets $\mathfrak{N}^{\prime}<\mathfrak{N}$ and Lemma 3 fails.

Let $\lim$ be a function on a subclass $\mathcal{C}$ of $\mathfrak{X}$ to $\mathfrak{X}$. For the purpose of this paper only we shall call $\{\mathfrak{C}, \lim \}$ a convergence class in $\mathfrak{X}$ if $\mathfrak{C}$ is not empty and if the following conditions are satisfied:

(a1) If $\mathfrak{N} \in \mathbb{C}$ and if $\mathfrak{N}^{\prime}<\mathfrak{N}$ then $\mathfrak{N}^{\prime} \in \mathbb{C}$.

(a2) If $\mathfrak{N} \in \mathfrak{C}, \mathfrak{N}^{\prime}<\mathfrak{N}$ and $\mathfrak{N}^{\prime} \in \mathcal{C}$ then $\lim \mathfrak{N}=\lim \mathfrak{N}^{\prime}$.

We shall refer to (a1) and (a2) together as condition (a). In what follows, $\{\mathfrak{e}, \lim \}$ shall be a convergence class in $\mathfrak{X}$. We shall also say that a net $\mathfrak{N} \in \mathcal{C}$ converges to $\lim \mathfrak{N}$.

Theorem 1. $\lim \mathfrak{N}=\lim$ inf $\mathfrak{N}$ for all $\mathfrak{N} \in \mathbb{C}$ iff $\lim \mathfrak{N}=\lim \sup \mathfrak{N}$ for all $\mathfrak{N} \in \mathbb{C}$.

Proof. Suppose $\lim \mathfrak{N}=\lim$ inf $\mathfrak{N}$ for all $\mathfrak{N} \in \mathbb{C}$ and fix a net $\mathfrak{N} \in \mathbb{C}$. By condition (a) we have $\lim \mathfrak{N}^{\prime}=\lim \mathfrak{N}$ for all $\mathfrak{N}^{\prime}<\mathfrak{N}$. By Lemma 3 and by our hypothesis we conclude $\lim \sup \mathfrak{N}=U_{\mathfrak{N}^{\prime}<\mathfrak{N}} \lim$ inf $\mathfrak{R}^{\prime}$ $=\bigcup_{\mathfrak{N}^{\prime}<\mathfrak{N}} \lim \mathfrak{N}^{\prime}=\lim \mathfrak{N}$. An analogous reasoning completes the proof in the other direction.

Theorem 1 may fail to hold if $\{\mathfrak{e}, \lim \}$ is not required to satisfy condition (a). Let $X$ be arbitrary but nonvoid, let $\mathfrak{X}=\exp X$ and let $\mathfrak{C}=\mathfrak{X}$. Define $\lim \mathfrak{N}=\lim$ inf $\mathfrak{N}$ for all $\mathfrak{N} \in \mathcal{C}$. Then $(\mathrm{a} 1)$ holds but (a2) and Theorem 1 fail to hold, as can be seen by considering the sequence $\mathfrak{N}=\{0, X, 0, X, \cdots\}$. On the other hand, let $\mathfrak{e}$ be the class of all nets frequently in $\{0\}$. Define $\lim \mathfrak{N}=0$ for all $\mathfrak{N} \in \mathbb{C}$. Then (a2) holds, but (a1) and Theorem 1 fail to hold, as is again demonstrated by the above example.

For the sake of brevity we shall call a convergence class $\{\mathfrak{e}, \lim \}$ in $\mathfrak{X}$ compact, if every net $\mathfrak{N} \in \mathbb{X}$ contains a converging subnet. For every $\mathfrak{N} \in \mathfrak{X}$ let $\mathfrak{S}(\mathfrak{N})=\left\{\lim \mathfrak{N}^{\prime}: \mathfrak{N}^{\prime} \in \mathcal{C}, \mathfrak{N}^{\prime}<\mathfrak{N}\right\}$.

THEOREM 2. Let $\{\mathfrak{C}, \lim \}$ be a compact convergence class in $\mathfrak{X}$ satisfying $\lim \mathfrak{N}=\lim$ inf $\mathfrak{N}=\lim$ sup $\mathfrak{N}$ for all $\mathfrak{N} \in \mathcal{C}$. Then, for all $\mathfrak{N} \in \mathfrak{X}$, 
$\lim \inf \mathfrak{R}=\cap \mathfrak{S}(\mathfrak{R})$ and lim sup $\mathfrak{N}=\bigcup \mathfrak{S}(\mathfrak{R})$.

Proof. Because of compactness and our hypothesis, we have lim sup $\mathfrak{N} \supset \lim \mathfrak{N}^{\prime}$ and lim inf $\mathfrak{N} \subset \lim \mathfrak{N}^{\prime}$ for every $\mathfrak{N} \in \mathfrak{X}$ and for some $\mathfrak{R}^{\prime}<\mathfrak{R}, \mathfrak{N}^{\prime} \in \mathcal{C}$. Therefore by Lemma 3

$$
\begin{aligned}
\lim \inf \mathfrak{N} & =\bigcap_{\mathfrak{N}^{\prime}<\mathfrak{N}} \lim \sup \mathfrak{N}^{\prime}=\bigcap_{\mathfrak{N}^{\prime}<\mathfrak{N}_{;} \mathfrak{N}^{\prime} \in \mathbb{C}} \lim \mathfrak{N}^{\prime}=\cap \mathfrak{S}(\mathfrak{R}) \\
\lim \sup \mathfrak{N} & =\bigcup_{\mathfrak{N}^{\prime}<\mathfrak{N}} \lim \inf \mathfrak{N}^{\prime}=\bigcup_{\mathfrak{N}^{\prime}<\mathfrak{N}_{;} \mathfrak{N}^{\prime} \in \mathfrak{C}} \lim \mathfrak{N}^{\prime}=U \mathfrak{S}(\mathfrak{R}) .
\end{aligned}
$$

Theorem 2 is false in this general form without the assumption of compactness of the convergence class $\{e, \lim \}$. Let $X$ be a countably infinite set with the discrete topology and let $\mathfrak{X}=\exp X$. Furthermore, let $\mathfrak{C}$ be the class of all constant nets in $\mathfrak{X}$ and define $\lim \mathfrak{N}=\lim$ inf $\mathfrak{R}$ $=\lim \sup \mathfrak{R}$ for all $\mathfrak{R} \in \mathbb{C}$. Then $\{\mathfrak{e}, \lim \}$ is a noncompact convergence class in $\mathfrak{X}$ satisfying the other hypothesis in Theorem 2 . Let $\mathfrak{R}$ be the sequence consisting alternatingly of the sets $\left\{x_{1}\right\}$ and $\left\{x_{2}, x_{i}\right\} \quad(i=3,4,5, \cdots)$. Then we have $0=\lim \inf \mathfrak{N} \neq \cap \mathfrak{S}(\mathfrak{N})$ $=\left\{x_{1}\right\}$ and $\left\{x_{1}, x_{2}\right\}=$ lim sup $\mathfrak{R} \neq \cup \mathfrak{S}(\mathfrak{R})=\left\{x_{1}\right\}$.

We note that under the hypotheses of Theorem 2 for every $N \in \mathfrak{X}$ there is at least one constant net converging to $N$. The following converse of Theorem 2 is obvious.

TheOREM 3. Let $\{\mathfrak{e}, \lim \}$ be a convergence class in $\mathfrak{X}$. If $\lim$ inf $\mathfrak{R}$ $=\cap \mathfrak{S}(\mathfrak{N})$ or if $\lim \sup \mathfrak{N}=\cup \mathfrak{S}(\mathfrak{R})$ for all $\mathfrak{R} \in \mathfrak{X}$, then $\lim \mathfrak{N}=\lim$ inf $\mathfrak{N}$ $=\lim \sup \mathfrak{R}$ for all $\mathfrak{R} \in \mathbb{C}$.

We note that under the hypothesis of Theorem 3 the convergence class $\{\mathfrak{C}, \lim \}$ is "almost" compact, i.e. if $\lim$ inf $\mathfrak{N}=\cap \mathfrak{C}(\mathfrak{R})$ for all $\mathfrak{N} \in \mathfrak{X}$, then every net $\mathfrak{N}$ for which lim inf $\mathfrak{N} \neq X$ must contain at least one converging subnet. An analogous statement applies in the dual case. Combining the Theorems 1, 2 and 3 we get the following corollary:

Corollary. Let $\{\mathfrak{e}, \lim \}$ be a compact convergence class in $¥$. Then the following statements are equivalent:

(1) $\lim \mathfrak{N}=\lim$ inf $\mathfrak{N}$ for all $\mathfrak{N} \in \mathcal{C}$.

(2) $\lim \mathfrak{R}=\lim \sup \mathfrak{\Re}$ for all $\mathfrak{R} \in \mathbb{C}$.

(3) lim inf $\mathfrak{N}=\cap \mathfrak{S}(\mathfrak{N})$ for all $\mathfrak{N} \in \mathfrak{x}$.

(4) lim sup $\mathfrak{R}=\bigcup \mathfrak{C}(\mathfrak{R})$ for all $\mathfrak{R} \in \mathfrak{X}$.

In general, the equation lim inf $\mathfrak{N}=\lim$ sup $\mathfrak{N}$ does not characterize nets belonging to a compact convergence class $\{\mathfrak{e}, \lim \}$ in $\mathfrak{X}$ even if $\lim \mathfrak{R}=\lim$ inf $\mathfrak{N}=\lim \sup \mathfrak{R}$ for all $\mathfrak{N} \in \mathbb{e}$ is supposed. Consider a nonvoid finite discrete space $X$. Let $\mathfrak{X}=\exp X$ and define $\mathcal{e}$ to be 
the class of all constant nets in $\mathfrak{X}$ and $\lim \mathfrak{R}=\lim$ inf $\mathfrak{N}=\lim \sup \mathfrak{R}$ for all $\mathfrak{R} \in \mathcal{C}$. Then $\{\mathfrak{C}, \lim \}$ is a compact convergence class in $\mathfrak{X}$. However, the sequence $\{X, 0,0, \cdots\}$ does not belong to $\mathcal{C}$. The situation changes immediately if we impose (as is usually done) one more condition on the convergence class $\{\mathfrak{e}, \lim \}$.

THEOREM 4. Let $\{\mathfrak{C}, \lim \}$ be a compact convergence class satisfying the following condition:

(b) If $\mathfrak{R} \in \mathfrak{X}$ does not converge to $N$ then there is an $\mathfrak{N}^{\prime}<\mathfrak{N}$ such that $\lim \mathfrak{N}^{\prime \prime} \neq N$ for all $\mathfrak{N}^{\prime \prime} \in \mathfrak{C}, \mathfrak{N}^{\prime \prime}<\mathfrak{N}^{\prime}$.

If any of the four statements of the corollary holds for $\{\mathfrak{C}, \lim \}$ then $\mathfrak{C}=\{\mathfrak{R} \in \mathfrak{X}: \lim$ inf $\mathfrak{N}=\lim \sup \mathfrak{N}\}$.

Proof. Let $\mathfrak{N} \in \mathfrak{X}$ and $\lim$ inf $\mathfrak{N}=\lim \sup \mathfrak{N}=N$. Then, by the corollary, $\lim \mathfrak{N}^{\prime \prime}=\lim$ inf $\mathfrak{N}^{\prime \prime}=\lim \sup \mathfrak{N}^{\prime \prime}=N$ for all $\mathfrak{N}^{\prime \prime} \in \mathfrak{C}$, $\mathfrak{N}^{\prime \prime}<\mathfrak{N}$. Because of condition (b), the net $\mathfrak{N}$ must converge to $N$.

As a trivial consequence, a compact convergence class $\{\mathfrak{C}, \lim \}$ in $\mathfrak{X}$, satisfying condition (b) and one of the four conditions stated in the corollary, has also the other property usually required for convergence classes [5, p. 74]: Each constant net converges to the constant.

Theorem 4 is in some sense a converse to the following result of Frolík [1, Theorems 2.7 and 2.14]:

Let $X$ be a regular topological space and let $\mathfrak{X}=\exp X$. If $\mathfrak{C}=\{\mathfrak{N} \in \mathfrak{X}: \lim$ inf $\mathfrak{N}=\lim \sup \mathfrak{N}\}$ and if $\lim \mathfrak{N}=\lim$ inf $\mathfrak{N}=\lim \sup \mathfrak{N}$ for all $\mathfrak{N} \in \mathfrak{C}$, then $\{\mathfrak{e}, \lim \}$ is a compact convergence class in $\mathfrak{X}$ satisfying condition (b).

Therefore, in particular, the statements 3 and 4 of the corollary are valid in this case. The hypotheses of Theorem 4 are also satisfied if $\mathfrak{X}$ is a compact Hausdorff space in some topology and if $\{\mathfrak{e}, \lim \}$ is the convergence class of convergent nets in this topological space. Applications of the above results to this special situation were implicit in $[2, \S 5]$ and $[3, \S 2]$ where the author was concerned with a topology in the set of all closed (normal) subgroups of a compact topological group.

\section{REFERENCES}

1. Zdeněk Frolikk, Concerning topological convergence of sets, Czechoslovak Math. J. 10 (85) (1960), 168-180.

2. Gilbert Helmberg, Zerlegungen des Mittelwertes fastperiodischer Funktionen. II, J. Reine Angew. Math. 208 (1961), 1-21.

3. - Topologische Untergruppenräume, J. Reine Angew. Math. 208 (1961), 164-180.

4. S. Mrovka, On the convergence of nets of sets, Fund. Math. 45 (1958), 237-246.

5. J. L. Kelley, General topology, Van Nostrand, New York, 1955.

UnIVERSITÄT INNSBRUCK, AUSTRIA 